\title{
CD40L mediated alternative NFKB-signaling induces resistance to BCR-inhibitors in patients with mantle cell lymphoma
}

\author{
Hilka Rauert-Wunderlich', Martina Rudelius ${ }^{1,2}$, Ingolf Berberich ${ }^{3}$ and Andreas Rosenwald ${ }^{1}$
}

\begin{abstract}
Drug resistance is a significant obstacle in cancer treatment and therefore a frequent subject of research. Developed or primary resistance limits the treatment success of inhibitors of the $B$ cell receptor (BCR) pathway in mantle cell lymphoma (MCL) patients. Recent research has highlighted the role of the nuclear factor-kappa B (NFkB) pathway in the context of resistance to BCR inhibitors in MCL. In this study, we analyzed the dependency of MCL cell lines on NFKB signaling and illustrated the ability of CD4OL to activate the alternative NFKB pathway in MCL. This activation leads to independency of classical NFKB signaling and results in resistance to BCR inhibitors. Therefore, ligands (such as CD40L) and their activation of the alternative NFKB pathway have a major impact on the drug response in MCL. Furthermore, this study indicates a protective role for cells expressing specific ligands as microenvironmental niches for MCL cells and underlines the significance of therapeutically targeting alternative NFKB signaling in MCL.
\end{abstract}

\section{Introduction}

Mantle cell lymphoma $(\mathrm{MCL})$ is a rare $\mathrm{B}$ cell nonHodgkin lymphoma characterized by a $\mathrm{t}(11 ; 14)(\mathrm{q} 13 ; \mathrm{q} 32)$ translocation, which leads to cyclin D1 overexpression ${ }^{1,2}$ and cell cycle deregulation ${ }^{3}$. In the past few years, advances have been made in treating MCL patients by targeting the $\mathrm{B}$ cell receptor (BCR) pathway with ibrutinib ${ }^{4}$. Bruton's tyrosine kinase (BTK) inhibitor occupies the active site of BTK and therefore blocks BCR signaling $^{5}$, which is essential to malignant B cells ${ }^{6}$. Unfortunately, some MCL patients show primary resistance to ibrutinib or develop secondary resistance after treatment. The reasons for primary resistance in patients are widely unknown, whereas for secondary resistance, Chiron et al. identified a C481S mutation at the ibrutinib binding site of $\mathrm{BTK}^{7}$. Although novel second-generation BTK

\footnotetext{
Correspondence: Hilka Rauert-Wunderlich (hilka.rauert-wunderlich@uniwuerzburg.de)

${ }^{1}$ Institute of Pathology, University of Würzburg and Comprehensive Cancer Center (CCC) Mainfranken, Würzburg, Germany

${ }^{2}$ Institute of Pathology, University of Düsseldorf, Düsseldorf, Germany

Full list of author information is available at the end of the article

Edited by M. Herold
}

(C) The Author(s) 2018 inhibitors are being evaluated ${ }^{8}$, understanding the reasons for primary resistance and further deciphering the molecular pathology of MCL is an important topic in research.

Rahal et al. showed that some MCL cell lines resistant to the BCR inhibitors ibrutinib and sotrastaurin have mutations in players of the alternative nuclear factor-kappa $B$ (NFkB) pathway. These mutations lead to activation of alternative NFKB signaling and identify an MCL subgroup that is independent of BCR signaling ${ }^{9}$. This mechanism of resistance highlights the importance of $B C R$ and $N F \kappa B$ signaling in the pathogenesis of $\mathrm{MCL}^{10}$.

Drug resistance is a significant obstacle in the treatment of cancer patients, and microenvironmental signaling often plays a crucial role by providing individual niches for cancer cells ${ }^{11}$. Recently, this role of microenvironmental effects was also described in $\mathrm{MCL}^{12-14}$. Apart from the mentioned mutations, microenvironmental signaling can also cause activation of the alternative NFkB pathway. Therefore, we questioned whether microenvironmental activation of the alternative $\mathrm{NF \kappa B}$ pathway can lead to BCR inhibitor resistance in MCL. An important ligand in microenvironmental signaling in lymphomas is tumor

\footnotetext{
(c) Open Access This article is licensed under a Creative Commons Attribution 4.0 International License, which permits use, sharing, adaptation, distribution and reproduction changes were made. The images or other third party material in this article are included in the article's Creative Commons license, unless indicated otherwise in a credit line to the material. If material is not included in the article's Creative Commons license and your intended use is not permitted by statutory regulation or exceeds the permitted use, you will need to obtain
} permission directly from the copyright holder. To view a copy of this license, visit http://creativecommons.org/licenses/by/4.0/. 

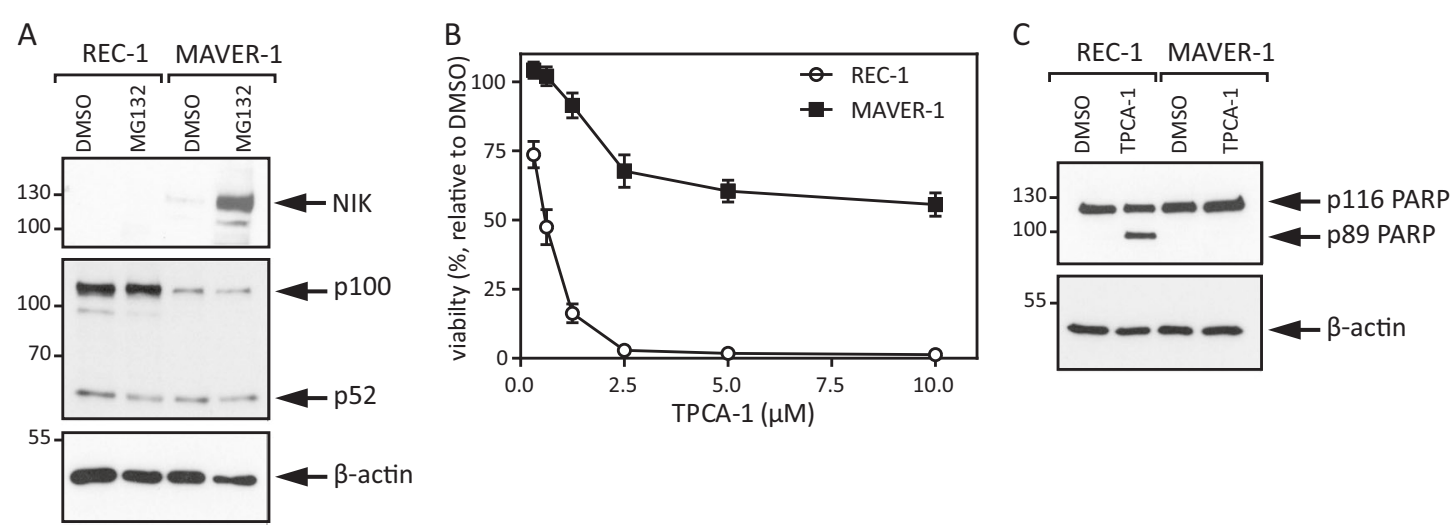

Fig. 1 REC-1 and MAVER-1 cells show distinct dependencies on classical NFKB signaling. a MCL cell lines were treated with MG132 (20 $\mu M)$ for $8 \mathrm{~h}$, and whole cell lysates were analyzed by Western blot. b REC-1 and MAVER-1 cells were incubated with increasing concentrations of TPCA-1 for $48 \mathrm{~h}$, and viability was determined via MTT assay. $\mathbf{c}$ Whole cell lysates of the MCL cell lines were prepared after treatment with TPCA-1 (5 $\mu \mathrm{M})$ for $24 \mathrm{~h}$ and were subsequently analyzed by Western blot

necrosis factor (TNF) ligand superfamily member 5 $(\mathrm{CD} 40 \mathrm{~L})^{15,16}$. CD40L belongs to the TNF ligand superfamily, binds to TNF receptor superfamily member 5 (CD40), and has a major role in B cell proliferation and differentiation ${ }^{17}$ as well as an effect on lymphomagenesis $^{18}$. CD40L can activate both the classical and the alternative NFKB pathways ${ }^{19,20}$. Activation of the classical $\mathrm{NF \kappa B}$ pathway, induced by the binding of a ligand to its receptor, leads to activation of the IKB $\alpha$-kinase (IKK) complex, which is composed of NFкB essential modifier (NEMO), IKK- $\alpha$ (IKK1), and IKK- $\beta$ (IKK2). This active complex then phosphorylates inhibitory I $\mathrm{B}$ proteins or the I $\mathrm{KB}$ domain (functioning as I $\kappa \mathrm{B}$ proteins) containing precursors, leading to their proteasomal degradation. ІкB proteins restrain $\mathrm{NF \kappa B}$ transcription factor dimers in the cytoplasm, and their degradation leads to the translocation of the transcription factor to the nucleus ${ }^{21-23}$.

Activation of the alternative $\mathrm{NF \kappa B}$ pathway by a ligand results in the accumulation of mitogen-activated protein kinase kinase kinase 14 (NIK) and the subsequent phosphorylation of NFKB subunit 2 (p100) by IKK1. This phosphorylation activates NFkB subunit 2 (p52) and V-Rel avian reticuloendotheliosis viral oncogene homolog B (RelB)containing NFKB dimers and allows their translocation to the nucleus ${ }^{21-23}$. TNF receptor-associated factor (TRAF) proteins also play a major role in NFKB signaling, and TRAF2 is necessary for classical NFkB pathway activation. TRAF2, together with TRAF3, shows inhibitory functions on alternative NFKB pathway activation by forming a complex with cellular inhibitors of apoptosis, leading to the ubiquitination and proteasomal degradation of $\mathrm{NIK}^{23}$. Interestingly, aberrant alternative $\mathrm{NFKB}$ signaling reportedly contributes to the development of lymphoid malignancies ${ }^{24}$.

The MCL cell line MAVER-1 harbors a biallelic TRAF3 deletion, leading to accelerated activation of the alternative
NFкB pathway ${ }^{9}$. We and others have previously shown the sensitivity of REC-1 cells to BCR inhibitors ${ }^{9,25}$. In this study, we therefore compared the effects of CD40Lmediated signaling in REC-1 and MAVER-1 cells.

\section{Results}

MCL cell lines with genetic lesions causing elevated alternative NFKB pathway activity are less dependent on IKK2-mediated signaling

To analyze the effect of the TRAF3 mutation in MAVER1 cells on the activity of the alternative NFkB pathway, we treated MCL cells with the proteasome inhibitor MG132 and detected higher levels of NIK in comparison to REC-1 cells (Fig. 1a). In addition, we detected an already higher basal level of processed p52 in relation to p100 levels in MAVER-1 cells than in REC-1 cells. This result reveals higher basal activity of the alternative NFKB pathway in MAVER-1 than in REC-1 cells. To further decipher the dependency of MCL cells on NFKB signaling, we challenged the cells with the IKK2-specific inhibitor TPCA-1, which inhibits classical NFKB signaling ${ }^{26,27}$. MAVER-1 cells showed only minor viability effects of TPCA-1 treatment compared to REC-1 cells, and Western blot analysis of whole cell lysates confirmed the resistance of MAVER-1 cells to TPCA-1 treatment (Fig. 1b, c). The precise inactivation of the classical NFkB pathway by TPCA-1 and the resistance of MAVER- 1 cells show that these cells have, to some extent, overcome the described dependency of MCL on classical NFkB signaling?.

Ligand stimulation activates the alternative NFKB pathway and rescues REC-1 cells from IKK2 inhibition-mediated toxicity

To show the effect of CD40L stimulation in MCL cell lines on the activity of the alternative NFKB pathway, we 

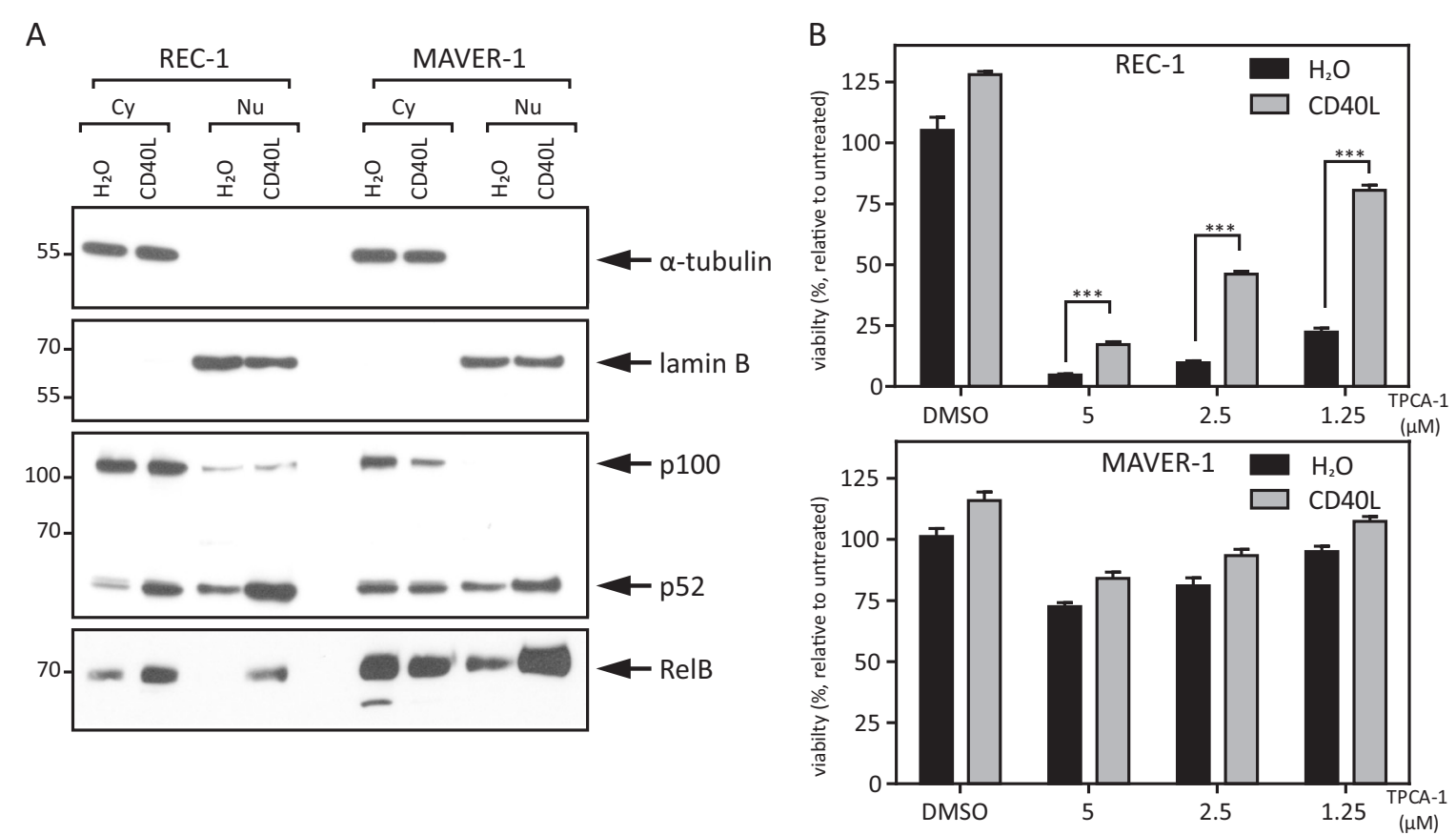

Fig. 2 CD40L rescues IKK2 inhibition-mediated toxicity by activating the alternative NFKB pathway. a REC-1 and MAVER-1 cells were stimulated with $\mathrm{CD} 40 \mathrm{~L}(100 \mathrm{ng} / \mathrm{ml})$ or $\mathrm{H}_{2} \mathrm{O}$ as a control for $18 \mathrm{~h}$, and cytoplasmic (Cy) and nuclear ( $\left.\mathrm{Nu}\right)$ protein fractions were analyzed by Western blot. a-Tubulin and lamin B served as controls for the purity of the individual cell compartment fractions. $\mathbf{b}$ MCL cell lines were stimulated with CD40L $(100 \mathrm{ng} / \mathrm{ml})$ or $\mathrm{H}_{2} \mathrm{O}$ as a control overnight followed by TPCA-1 treatment at the indicated concentration. After an additional $24 \mathrm{~h}$, viability was determined by MTT assay $\left({ }^{*} p<0.001 ;{ }^{* *} p<0.0001 ;{ }^{* * *} p<0.00001\right)$

treated REC-1 and MAVER-1 cells with CD40L overnight and analyzed the cellular localization of NFKB transcription factors. CD40L treatment caused accelerated p100 processing to $\mathrm{p} 52$ and a higher expression of the active transcription factors p52 and RelB in the nucleus in REC1 cells. This effect was also detectable in MAVER-1 cells, but to a lesser extent (Fig. 2a). These data reveal and underline the described ability of CD40L to activate the alternative NFKB pathway in MCL cells ${ }^{20}$.

The cell line REC-1 showed a high sensitivity towards treatment with the IKK2-specific inhibitor TPCA-1 (Fig. 1b, c and 2b), yet prestimulation with CD40L significantly rescued REC-1 cells from TPCA-1-induced cell death (Fig. 2b). This effect was considerable compared to the basal enhanced proliferation after CD40L prestimulation in the control group. In the TPCA-1-resistant MAVER-1 cell line, CD40L also slightly enhanced proliferation but with no significant effect. Taken together, these results illustrate the ability of CD40L to activate the alternative NFKB pathway in MCL cell lines and to substitute the dependency on classical NFKB signaling in REC-1 cells through the activity of alternative NFKB signaling. Chiron et al. argued for a functional role of the anti-apoptotic protein $\mathrm{Bcl}-\mathrm{xL}$ in the context of CD40 stimulation in some MCL cell lines ${ }^{20,28}$. We measured the Bcl-xL expression level and show in
Supplementary Figure 1 that there was no major difference in Bcl-xL expression in REC-1 cells. However, compared to MAVER-1 cells, REC-1 cells showed a very high basal Bcl-xL expression level. In MAVER-1 cells, we observed faint induction of $\mathrm{Bcl}-\mathrm{xL}$, as described by Chiron et al. which was inhibited by TPCA-1. Therefore, in this cell line, the classical NFKB pathway regulates Bcl-xL, and the external stimulation with $\mathrm{CD} 40 \mathrm{~L}$ does not induce higher expression of this anti-apoptotic protein in REC-1 cells, most likely due to the already high basal activity of this pathway.

\section{CD40L-mediated alternative NFKB signaling in MCL cell lines is independent of the BCR pathway}

To decipher the potential effect of this signaling pathway on drug resistance, we prestimulated the BCR inhibitorsensitive cell line REC-1 and the inhibitor-resistant cell line MAVER-1 with CD40L overnight to activate the alternative NFKB pathway. Subsequently, the cells were treated with two inhibitors: ibrutinib or sotrastaurin. Western blot analysis of cytoplasmic and nuclear protein fractions revealed that $\mathrm{CD} 40 \mathrm{~L}$-induced $\mathrm{p} 100$ processing to p52 and its nuclear translocation were influenced neither by the PKC inhibitor sotrastaurin nor by the BTK inhibitor ibrutinib (Fig. 3a, b). The same effect was detectable for the enhanced expression and nuclear levels of RelB after 
CD40L treatment in REC-1 cells. In MAVER-1 cells, this effect on RelB was also obvious, whereas the effect on p100/p52 was preeminent in REC-1 cells. This difference might be due to the higher basal levels of the alternative NFkB pathway activity in MAVER-1 cells. Furthermore, CD40L treatment had no major effect on the NFkB transcription factors p50 and p65 (Fig. 3a, b), which are regulated by the classical $\mathrm{NF \kappa B}$ pathway ${ }^{27}$. This result indicates a more prominent role of CD40L on the alternative than on the classical NFKB pathway in this experimental setting. Additionally, L-929 cells were transiently transfected with a CD40L expression plasmid and co-

A
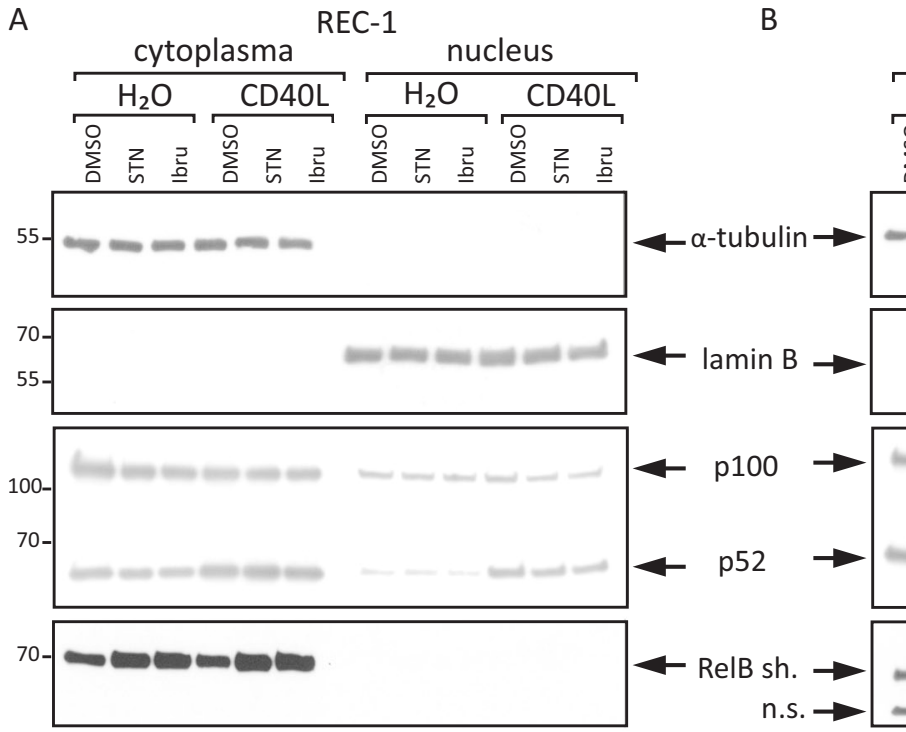

RelB sh.

n.s. $\rightarrow$
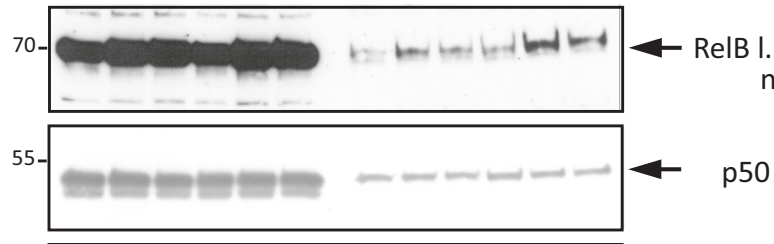

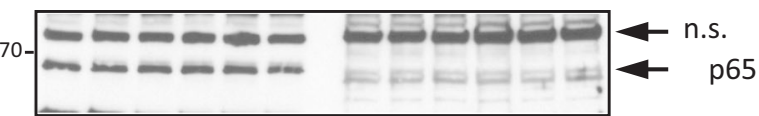
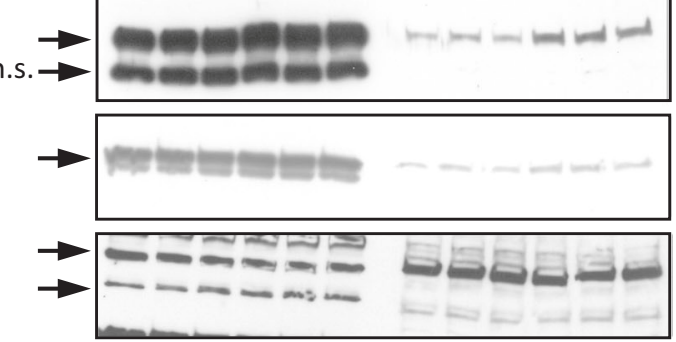

C

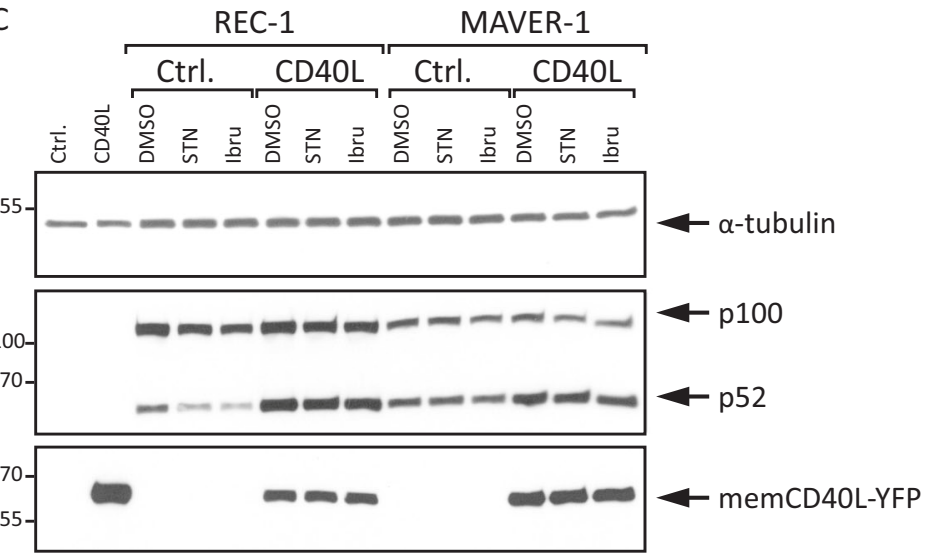

Fig. 3 CD40L activates alternative NFKB signaling independently of the BCR pathway. The MCL cell lines REC-1 a and MAVER-1 b were preincubated with CD40L (100 ng/ml) or $\mathrm{H}_{2} \mathrm{O}$ for $18 \mathrm{~h}$ and subsequently treated with either sotrastaurin (STN, $\left.3 \mu \mathrm{M}\right)$, ibrutinib (Ibru, $\left.400 \mathrm{nM}\right)$ or DMSO as a control for an additional $24 \mathrm{~h}$. Cytoplasmic and nuclear protein extracts were analyzed by Western blot for the indicated proteins; "sh." stands for a short and "I." for a long exposure time for RelB detection. c L-929 cells were transfected with a CD40L expression plasmid or a control plasmid and co-cultured with REC-1 and MAVER-1 cells for $18 \mathrm{~h}$. Subsequently, the cells were treated with sotrastaurin ( $3 \mu \mathrm{M})$, ibrutinib (400 nM) or DMSO for an additional $24 \mathrm{~h}$, and whole cell lysates were analyzed by Western blot 
cultivated with REC-1 and MAVER-1 cells (Fig. 3c). This microenvironment-mimicking experiment showed similar results as stimulation with recombinant $\mathrm{CD} 40 \mathrm{~L}$. The induction of $\mathrm{p} 100$ processing to $\mathrm{p} 52$ was not influenced by inhibition of the BCR pathway. Therefore, both cell lines responded to $\mathrm{CD} 40$ stimulation with accelerated activity of the alternative NFkB pathway, and this signaling was neither influenced by the BTK inhibitor ibrutinib nor by the PKC inhibitor sotrastaurin.

\section{Activation of the alternative NFKB pathway by recombinant $C D 40 L$ induces resistance to $B C R$ inhibitors in REC-1 cells}

Activation of the alternative NFkB pathway in MCL cell lines results in nuclear translocation of the transcription factors p52 and RelB (Fig. 3a, b). Although little is known specifically about CD40L-induced p52/RelB target genes, it is established that these transcription factors play a major role in B cell development, survival and homeostasis (reviewed in $^{29}$ ). Therefore, we questioned whether CD40L-induced signaling in MCL cells causes drug resistance. Viability assays revealed a protective effect of CD40L prestimulation on BCR inhibitor treatment (Fig. 4). Profoundly, this effect was detectable and significant in the sensitive cell line REC-1 for both inhibitors at clinically relevant concentrations (sotrastaurin $3 \mu \mathrm{M}$ : $p<0.0001$ and ibrutinib $400 \mathrm{nM}: p<0.0001)$ but also in the resistant cell line MAVER-1 at nonphysiologically high sotrastaurin concentrations $(27 \mu \mathrm{M}: \quad p<0.001)$ (Fig. 4a). These data argue for a protective role of CD40Lmediated alternative $\mathrm{NF \kappa B}$ signaling leading to drug resistance in MCL.

CD40 is known to activate also various other pathways than the NFKB pathways ${ }^{30}$. Therefore, we studied the effects of inhibitors of diverse pathways on the CD40L mediated resistance to the BCR inhibitors in REC-1 cells (Fig. 4c). Apart from NVP-BEZ 235, a dual PI3K and mTOR inhibitor, the other inhibitors (Akti-1/2, a selective inhibitor of Akt1 /Akt2; AZD8330 a selective MEK1/2 inhibitor; ERK inhibitor II, an inhibitor of ERK1 and ERK2; 2-Bromoaldisine, an inhibitor of the Raf/MEK-1/ MAPK cascade) alone show little toxicity. The combination of the inhibitors with BCR inhibitors showed only slight additive toxic effects. Interestingly, CD40L prestimulation rescued REC-1 cells from the sotrastaurin and ibrutinib mediated toxicity even in combination with the different inhibitors. Regarding Akti-1/2, AZD8330, ERK inhibitor II and 2-Bromoaldisine the rescue effect of CD40L is hardly influenced by the additional pathway inhibition and the rescue effect is still highly significant, with only one exception (ERK inhibitor II in combination with ibrutinib). Also, the REC-1 cells treated with NVPBEZ 235 are in part rescued from the toxicity induced by the BCR inhibitors. This supports the protective function of the alternative $\mathrm{NF \kappa B}$ pathway against $\mathrm{BCR}$ inhibitors in MCL cells.

\section{Discussion}

Targeting the BCR pathway is a new and promising approach to treat MCL, and ibrutinib as a clinically used drug targeting this pathway in MCL shows favorable response rates ${ }^{31,32}$. Unfortunately, subsequent studies have revealed that some patients develop resistance upon ibrutinib treatment and relapse ${ }^{7,33}$. Therefore, efforts have been made to develop second-generation BCR inhibitors and to decipher the formation of drug resistance in MCL. In our own and other studies, it was shown that the MAVER-1 cell line is resistant to the two inhibitors ibrutinib and sotrastaurin, whereas the cell line REC- 1 is sensitive $^{9,25}$. Rahal et al. further postulated an important role of the alternative NFKB pathway in BCR inhibitor resistance for MCL cells, as, for example, the resistant MAVER-1 cell line harbors a biallelic deletion of TRAF3 ${ }^{9}$. TRAF3 has an inhibitory function in regulating the alternative NFאB pathway ${ }^{34}$. Apart from specific mutations, microenvironmental factors can also influence the activity of the alternative NFkB pathway. Particularly, for MCL, stromal cells have been shown to activate this signaling pathway ${ }^{35}$. Therefore, we questioned the role of external signaling on the alternative $\mathrm{NFKB}$ pathway in $\mathrm{MCL}$ and its effect on drug responses.

Our data illustrate the high basal activity of the alternative NFKB pathway in MAVER-1 cells compared to that in REC-1 cells, most likely caused by the TRAF3 deletion in MAVER.1 cells. In addition, MAVER-1 cells show lower p100 levels than REC-1 cells, arguing not only for a higher activity of the alternative NFKB pathway but also for a lower activity of the basal classical NFKB signaling pathway, as p100 is a target of classical NFKB signaling ${ }^{22,27}$. Additionally, Supplementary Figure 1 illustrates the reduction of p100 levels in the context of CD40L treatment and additional inhibition of the classical pathway, underlining the activating role of $\mathrm{CD} 40 \mathrm{~L}$ on alternative NFKB signaling in MCL cells. Furthermore, MAVER-1 cells are less dependent on classical $\mathrm{NF} \kappa \mathrm{B}$ signaling, leading to the assumption that alternative NFKB signaling in part may substitute for classical signaling in MCL cells.

To analyze the microenvironmental effect on the activity of the alternative NFKB pathway in MCL, we chose to examine CD40L. All MCL cells, as other B cells, express its receptor $\mathrm{CD} 40^{36}$, and CD40L is expressed not only on $\mathrm{T}$ cells but also on other cell types ${ }^{37,38}$, which are common in the MCL microenvironment. Therefore, the CD40L-CD40 interaction and the CD40L-rich microenvironment play a part in the pathogenesis of $\mathrm{MCL}^{39}$. In addition, Chiron et al. previously described the role of CD40 stimulation on the activity of NFKB signaling in 


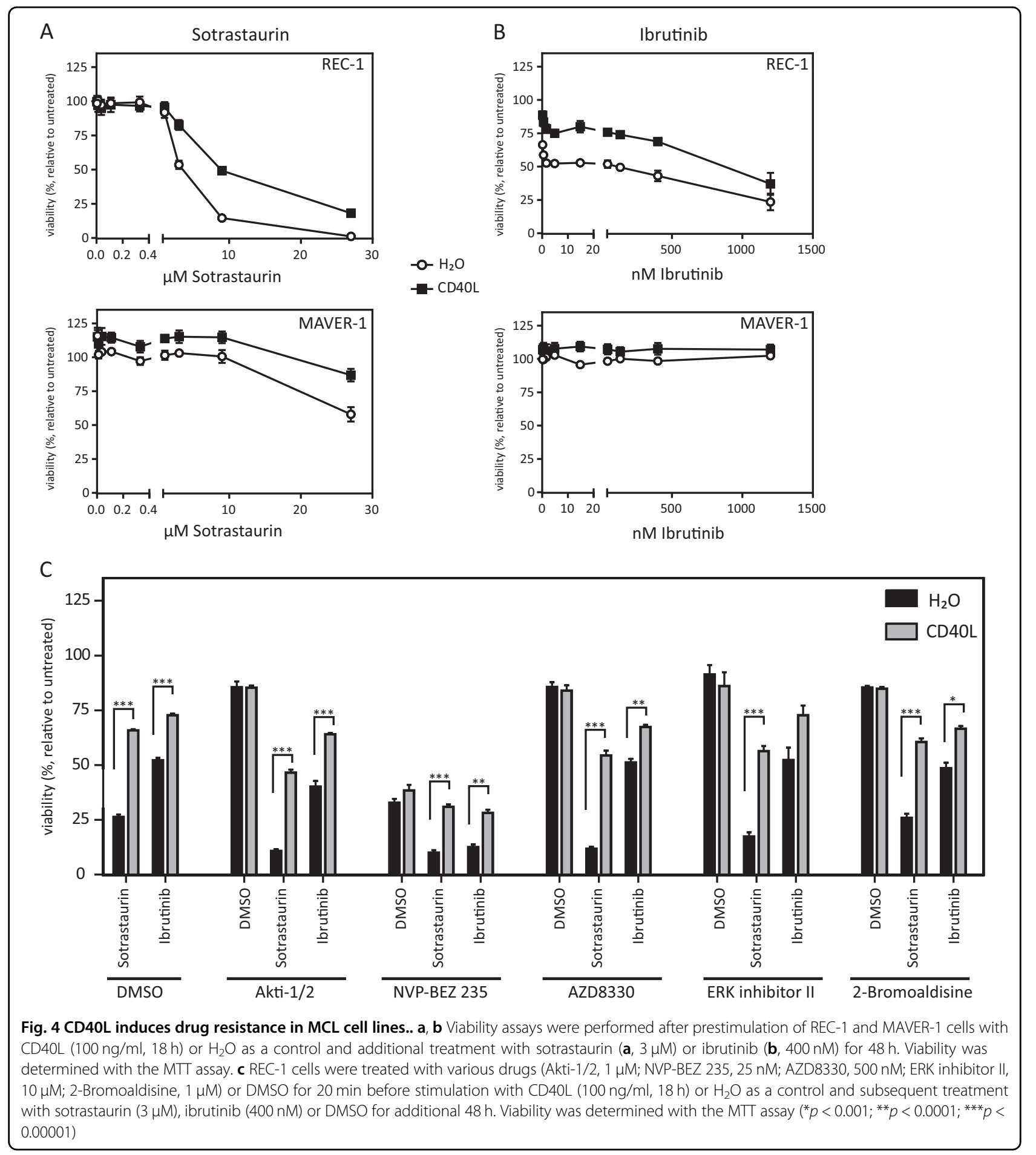

$\mathrm{MCL}^{20,28}$. In our experiments to stimulate $\mathrm{CD} 40$, we chose a highly active recombinant oligomer from Enzo, which mimics the in vivo-occurring membrane-assisted aggregation of CD40L. Our study showed a significant proliferative effect of CD40L stimulation on REC-1 cells and (to a lesser extent) on MAVER-1 cells. These data are contradictive to reports describing an antiproliferative effect of CD40L on MCL cell lines ${ }^{40}$ but are in line with other studies describing the CD40-system ${ }^{15,39}$. Furthermore, CD40L stimulation induced alternative NFkB pathway signaling in both cell lines. This activation rescued REC-1 cells from IKK2 inhibition-mediated toxicity 
and is therefore independent of signaling via CD40 on the classical NFKB pathway.

Because Rahal et al. previously postulated the possibility of activating mutations of alternative $\mathrm{NFKB}$ pathway members to substitute for dependency on BCR signaling, these data indicate the potential of microenvironmentally induced alternative $\mathrm{NF \kappa B}$ signaling to substitute for classical NFkB signaling in dependent cells.

The CD40L-mediated activity of the alternative NFKB pathway was obvious in both cell lines, as shown by enhanced nuclear p52 and RelB levels. Although MAVER1 cells possess higher basal levels of alternative NFKB signaling activity due to TRAF3 deletion, external ligand stimulation could push this signaling to a higher level. However, in both cell lines the BCR inhibitory drugs sotrastaurin and ibrutinib had no major effect on the transcription factor p52, neither at the basal nor at the induced level. Only RelB levels were slightly enhanced after inhibitor treatment in REC-1 cells which might be caused by an unknown inhibition mediated feedback loop. Similar but even more robust effects regarding p100 processing to p52 were detectable in the microenvironmentmimicking co-culture experiment. In sum, these data indicate that alternative NFKB signaling pathway activity is independent of BCR signaling in MCL cells. With regard to viability, it was clear that $\mathrm{CD} 40 \mathrm{~L}$ stimulation protected REC-1 cells from BCR inhibitor-mediated toxicity, especially at clinically relevant concentrations, whereas no major effect was seen in the resistant cell line MAVER-1. This effect seems not to be dependent on the antiapoptotic protein Bcl-xL or induced classical NFKB signaling. Additionally, inhibition of various other pathways, which can be influenced by CD40 stimulation, had no impact on the CD40L mediated rescue of REC-1 cells and therefore supports the protective role of alternative NFkB pathway activity against BCR inhibitors in MCL.

We also analyzed the ability of TNF ligand superfamily member 13b (BAFF) to activate the alternative NFKB pathway in the studied MCL cell lines. Unfortunately, stimulation with BAFF did not robustly induce p100 processing to p52 nor did it clearly rescue REC-1 cells from TPCA-1 or BCR inhibitors-mediated toxicity (data not shown). Experiments to generate REC-1 cells with a silenced alternative NFKB pathway in order to strengthen the functional role of this pathway in drug resistance were unsuccessful and further studies are needed to decipher the substantial role of the alternative NFKB pathway in MCL.

In conclusion, the data presented in this study argue for the protective potential of microenvironmentally mediated activation of the alternative NFKB pathway in MCL cells against BCR signaling-associated drugs, which might represent a physiologic niche for MCL relapse. Additionally, these data provide evidence for the potential of the alternative NFkB pathway as a possible therapeutic target in MCL.

\section{Materials and methods \\ Cell lines reagents and antibodies}

REC-1, MAVER-1, and L-929 cell lines were purchased from Deutsche Sammlung von Mikroorganismen und Zellkulturen (DSMZ, Braunschweig, Germany) and were cultured in RPMI1640 (Thermo Fisher Scientific, Waltham, MA, USA) with $10 \%$ (REC-1, L-929) or $20 \%$ (MAVER-1) heat-inactivated fetal bovine serum (PAN Biotech, Aidenbach, Germany and Thermo Fisher Scientific) supplemented with $2 \mathrm{mmol} / \mathrm{l}$ L-glutamine (PAN Biotech). All cell lines were cultured at $37^{\circ} \mathrm{C}$ with $5 \% \mathrm{CO}_{2}$ and were regularly tested for the absence of mycoplasma infection with the Venor GeM OneStep kit (Minerva Biolabs, Berlin, Germany).

MEGACD40L (referred to as CD40L) and 2Bromoaldisine were purchased from ENZO Life Sciences (Lörrach, Germany). MG132 was purchased from SigmaAldrich (Munich, Germany), and TPCA-1, ibrutinib and sotrastaurin were purchased from Selleck Chemicals (Absource Diagnostics, Munich, Germany). NVP-BEZ 235 was purchased from Hycultec (Beutelsbach, Germany) and AZD8330 was purchased from Biozol (Eching, Germany). Primary antibodies for PARP (\#9532), NIK (\#4994), $\beta$-actin (\#4970), CD40L (\#15094), Bcl-xL (\#54H6) and secondary horseradish peroxidase (HRP)-conjugated anti-rabbit IgGHRP antibody (\#7074) were purchased from Cell Signaling Technologies (Danvers, MA, USA). The primary antibodies for lamin B (\#sc-6216), p65 (\#sc-71677), p50 (\#sc-7178) and RelB (\#sc-226) were from Santa Cruz Biotechnology (Dallas, TX, USA). The p100/p52 (\#05-361) antibody, ERK inhibitor II and Akti-1/2 were purchased from Merck Millipore (Darmstadt, Germany), and $\alpha$-tubulin (DM1A) was purchased from Thermo Fisher Scientific. Secondary HRP-conjugated anti-mouse (\#P0260), anti-goat (\#P0160), and anti-rabbit (\#P0448) antibodies were purchased from Dako (Glostrup, Denmark).

\section{Viability assay}

Cells were seeded into 96-well plates and stimulated as indicated in the various figure legends. After the specific treatments, metabolic activity was measured with the cell proliferation kit I (3-(4,5-dimethylthiazol-2-yl)-2,5diphenyltetrazolium bromide; MTT) (Roche Diagnostics, Mannheim, Germany) in accordance with the manufacturer's instructions. Cell viability was calculated as the percentage of cells either treated with dimethyl sulfoxide (DMSO, Carl Roth, Karlsruhe, Germany) or untreated (100\%) and background staining in dead cells $(0 \%)$ was measured following treatment with $\mathrm{H}_{2} \mathrm{O}_{2}$ (Merck Millipore Darmstadt, Germany). DMSO treatment was performed in the equivalent dilution as the highest 
corresponding reagent dilution in the individual experiment, and the error bars represent $+/-$ standard error of the mean (SEM). Combined data are shown from two or three identical individual experiments.

\section{Co-culture with CD40L-expressing cells}

L-929 cells were transiently transfected with Lipofectamin3000 according to the manufacturer's instructions with a CD40L expression plasmid (human full-length CD40L-pEYFP-C1) or a control plasmid (pEYFP-C1) overnight. Prof. Dr. Harald Wajant kindly provided both plasmids. The next day, the cells were seeded and co-cultured with REC-1 and MAVER-1 cells. After $18 \mathrm{~h}$ of co-culture, the cells were treated with sotrastaurin, ibrutinib or DMSO as a control. After an additional $24 \mathrm{~h}$, the cells were harvested, and whole cell lysates were prepared. The transfection efficiency was verified by analyzing the membrane CD40L expression by Western blot.

\section{Western blot}

Cells were stimulated as indicated in the individual figure legends and subsequently washed with PBS. Nuclear and cytoplasmic protein fractions were isolated by use of the CelLytic NuCLEAR Extraction Kit from Sigma-Aldrich (Munich, Germany) according to the manufacturer's instructions. Whole cell lysates were prepared by lysing cell pellets for $20 \mathrm{~min}$ on ice in lysis buffer (20 mM Hepes; $350 \mathrm{mM} \mathrm{NaCl} ; 1 \mathrm{mM} \mathrm{MgCl}$; 0.5 mM EDTA, pH 8.0; and 0.1 mM EGTA, pH 8.0). After centrifugation, the protein concentrations were measured from the supernatant with the Bradford assay, and the lysates were supplemented with loading buffer (100 $\mathrm{mM}$ Tris; $\mathrm{pH}$ 6.8; $20 \%$ glycerol; $8 \%$ SDS; $10 \%$ betamercaptoethanol; and $0.01 \%$ bromophenol blue) and boiled to $96^{\circ} \mathrm{C}$ for $5 \mathrm{~min}$. Samples were then loaded for sodium dodecyl sulfate polyacrylamide gel electrophoresis, and the proteins were transferred to nitrocellulose membranes and checked by Ponceau S (SigmaAldrich) staining. Blots were subsequently incubated for $1 \mathrm{~h}$ with Tris-buffered saline containing $0.1 \%$ Tween 20 and $5 \%$ milk powder and incubated with primary and HRP-conjugated secondary antibodies. The antigenantibody complexes were visualized with SuperSignal West Pico Chemiluminescent Substrate (Thermo Fisher Scientific, Waltham, MA, USA) and Amersham Hyperfilm ECL (GE Healthcare, Freiburg, Germany). Different proteins were in part visualized on separate but identical blots.

\section{Statistical analysis}

Statistical analysis was performed using GraphPad Prism 6 (GraphPad Software, Inc., La Jolla, CA, USA) to analyze the significance of viability changes after CD40L prestimulation (Fig. 2b) with multiple $t$-tests (* $p<0.001$; $* p<0.0001 ; * * * 00.00001)$.

Acknowledgements

The authors thank Tina Grieb for excellent technical assistance.

\section{Author details}

${ }^{1}$ Institute of Pathology, University of Würzburg and Comprehensive Cancer Center (CCC) Mainfranken, Würzburg, Germany. ${ }^{2}$ Institute of Pathology, University of Düsseldorf, Düsseldorf, Germany. ${ }^{3}$ Institute of Virology and Immunobiology, University of Würzburg, Würzburg, Germany

\section{Conflict of interest}

The authors declare they have no conflict of interest.

\section{Publisher's note}

Springer Nature remains neutral with regard to jurisdictional claims in published maps and institutional affiliations.

\section{Supplementary information}

The online version of this article (https://doi.org/10.1038/s41419-017-0157-6) contains supplementary material.

Received: 18 November 2016 Revised: 30 October 2017 Accepted: 31 October 2017

Published online: 24 January 2018

\section{References}

1. Raffeld, M. \& Jaffe, E. S. bcl-1, t(11;14), and mantle cell-derived lymphomas. Blood 78, 259-263 (1991).

2. Williams, M. E. \& Swerdlow, S. H. Cyclin D1 overexpression in non-Hodgkin's lymphoma with chromosome 11 bcl-1 rearrangement. Ann. Oncol. 5, 71-73 (1994).

3. Jares, P., Colomer, D. \& Campo, E. Molecular pathogenesis of mantle cell lymphoma. J. Clin. Invest. 122, 3416-3423 (2012).

4. Wang, Y., Zhang, L. L., Champlin, R. E. \& Wang, M. L. Targeting Bruton's tyrosine kinase with ibrutinib in B-cell malignancies. Clin. Pharmacol. Ther. 97, 455-468 (2015).

5. Honigberg, L. A. et al. The Bruton tyrosine kinase inhibitor PCl-32765 blocks Bcell activation and is efficacious in models of autoimmune disease and B-cell malignancy. Proc. Natl Acad. Sci. USA 107, 13075-13080 (2010).

6. Buchner, M. \& Muschen, M. Targeting the B-cell receptor signaling pathway in B lymphoid malignancies. Curr. Opin. Hematol. 21, 341-349 (2014).

7. Chiron, D. et al. Cell-cycle reprogramming for PI3K inhibition overrides a relapse-specific C481S BTK mutation revealed by longitudinal functional genomics in mantle cell lymphoma. Cancer Discov. 4, 1022-1035 (2014).

8. Wu, J., Liu, C., Tsui, S. T. \& Liu, D. Second-generation inhibitors of Bruton tyrosine kinase. J. Hematol. Oncol. 9, 80 (2016).

9. Rahal, R. et al. Pharmacological and genomic profiling identifies NF-kappa Btargeted treatment strategies for mantle cell lymphoma. Nat. Med. 20, 87-92 (2014).

10. Colomer, D. \& Campo, E. Unlocking new therapeutic targets and resistance mechanisms in mantle cell lymphoma. Cancer Cell 25, 7-9 (2014).

11. Chen, F. et al. New horizons in tumor microenvironment biology: challenges and opportunities. BMC Med. 13, 45 (2015).

12. Kurtova, A. V., Tamayo, A. T., Ford, R. J. \& Burger, J. A. Mantle cell lymphoma cells express high levels of CXCR4, CXCR5, and VLA-4 (CD49d): importance for interactions with the stromal microenvironment and specific targeting. Blood 113, 4604-4613 (2009)

13. Burger, J. A. \& Ford, R. J. The microenvironment in mantle cell lymphoma: cellular and molecular pathways and emerging targeted therapies. Semin. Cancer Biol. 21, 308-312 (2011).

14. Jayappa, K. D. et al. Microenvironmental agonists generate de novo phenotypic resistance to combined ibrutinib plus venetoclax in CLL and MCL. Blood Adv. 1, 933-946 (2017).

15. Visser, H. P., Tewis, M., Willemze, R. \& Kluin-Nelemans, J. C. Mantle cell lymphoma proliferates upon IL-10 in the CD40 system. Leukemia 14, 1483-1489 (2000). 
16. Scott, D. W. \& Gascoyne, R. D. The tumour microenvironment in B cell lymphomas. Nat. Rev. Cancer 14, 517-534 (2014).

17. Baker, S. J. \& Reddy, E. P. Modulation of life and death by the TNF receptor superfamily. Oncogene 17, 3261-3270 (1998).

18. Hömig-Hölzel, C. et al. Constitutive CD40 signaling in B cells selectively activates the noncanonical NF-kappaB pathway and promotes lymphomagenesis. J. Exp. Med. 205, 1317-1329 (2008).

19. Elgueta, R. et al. Molecular mechanism and function of CD40/CD40L engagement in the immune system. Immunol. Rev. 229, 152-172 (2009).

20. Chiron, D. et al. Biological rational for sequential targeting of Bruton tyrosine kinase and $\mathrm{BCl}-2$ to overcome CD40-induced ABT-199 resistance in mantle cell lymphoma. Oncotarget 6, 8750-8759 (2015).

21. Scheidereit, C. IkappaB kinase complexes: gateways to NF-kappaB activation and transcription. Oncogene 25, 6685-6705 (2006).

22. Hoffmann, A., Natoli, G. \& Ghosh, G. Transcriptional regulation via the NFkappaB signaling module. Oncogene 25, 6706-6716 (2006).

23. Sun, S. C. \& Ley, S. C. New insights into NF-kappaB regulation and function. Trends Immunol. 29, 469-478 (2008).

24. Sun, S. C. Non-canonical NF-kappaB signaling pathway. Cell Res. 21, 71-85 (2011).

25. Rauert-Wunderlich, H., Rudelius, M., Ott, G. \& Rosenwald, A. Targeting protein kinase C in mantle cell lymphoma. Br. J. Haematol. 173, 394-403 (2016).

26. Rauert-Wunderlich, $\mathrm{H}$. et al. The IKK inhibitor Bay 11-7082 induces cell death independent from inhibition of activation of NFkappaB transcription factors. PLoS One 8, e59292 (2013).

27. Rauert, $H$. et al. Membrane tumor necrosis factor (TNF) induces p100 processing via TNF receptor-2 (TNFR2). J. Biol. Chem. 285, 7394-7404 (2010).

28. Chiron, D. et al. Rational targeted therapies to overcome microenvironmentdependent expansion of mantle cell lymphoma. Blood 128, 2808-2818 (2016)

29. Dejardin, E. The alternative NF-kappaB pathway from biochemistry to biology: pitfalls and promises for future drug development. Biochem. Pharmacol. 72 1161-1179 (2006)
30. So, T. \& Croft, M. Regulation of Pl-3-kinase and Akt signaling in T lymphocytes and other cells by TNFR family molecules. Front. Immunol. 4, 139 (2013).

31. Wang, M. L. et al. Targeting BTK with ibrutinib in relapsed or refractory mantlecell lymphoma. N. Engl. J. Med. 369, 507-516 (2013).

32. Advani, R. H. et al. Bruton tyrosine kinase inhibitor ibrutinib (PCl-32765) has significant activity in patients with relapsed/refractory B-cell malignancies. J. Clin. Oncol. 31, 88-94 (2013).

33. Martin, P. et al. Postibrutinib outcomes in patients with mantle cell lymphoma. Blood 127, 1559-1563 (2016).

34. Liao, G., Zhang, M., Harhaj, E. W. \& Sun, S. C. Regulation of the NF-kappaBinducing kinase by tumor necrosis factor receptor-associated factor 3-induced degradation. J. Biol. Chem. 279, 26243-26250 (2004).

35. Medina, D. J. et al. Mesenchymal stromal cells protect mantle cell lymphoma cells from spontaneous and drug-induced apoptosis through secretion of B-cell activating factor and activation of the canonical and non-canonical nuclear factor kappaB pathways. Haematologica 97, 1255-1263 (2012).

36. Castillo, R. et al. Proliferative response of mantle cell lymphoma cells stimulated by CD40 ligation and IL-4. Leukemia 14, 292-298 (2000).

37. Wykes, M. Why do B cells produce CD40 ligand? Immunol. Cell Biol. 81 328-331 (2003).

38. Angelico, F. et al. Enhanced soluble CD40L in patients with the metabolic syndrome: relationship with in vivo thrombin generation. Diabetologia 49, 1169-1174 (2006)

39. Andersen, N. S. et al. Soluble CD40 ligand induces selective proliferation of lymphoma cells in primary mantle cell lymphoma cell cultures. Blood $\mathbf{9 6}$ 2219-2225 (2000)

40. Jin, Z. et al. CD40 ligand stimulation inhibits the proliferation of mantle cell lymphoma lines. Anticancer Res. 24, 691-697 (2004). (2B). 